\title{
Cisplatin-Irinotecan Regimen
}

National Cancer Institute

\section{Source}

National Cancer Institute. Cisplatin-Irinotecan Regimen. NCI Thesaurus. Code C63414.

A regimen consisting of cisplatin and irinotecan used to treat advanced-stage non-small cell lung cancer. 